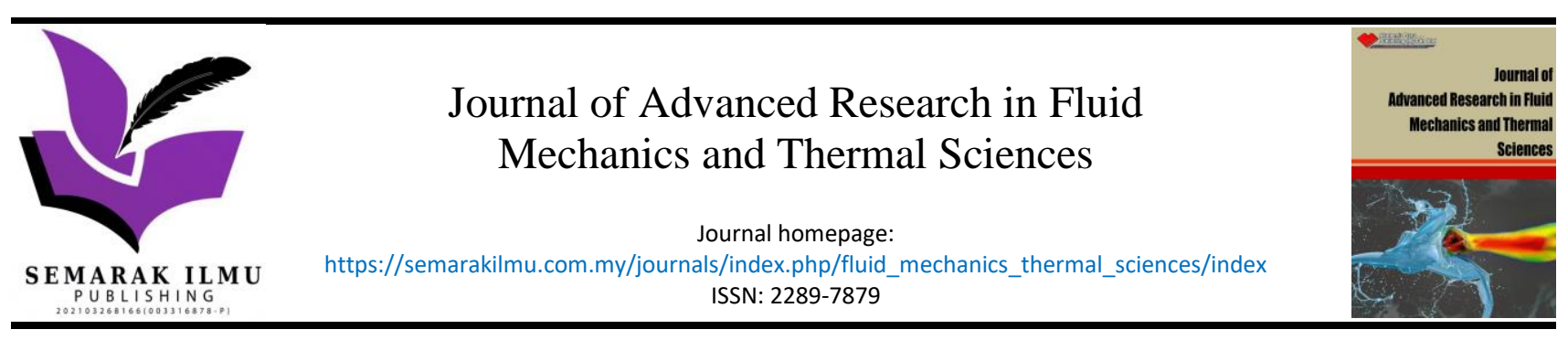

\title{
The Flow of An Inhomogeneous Fluid Inside a Sphere
}

\author{
M. M. Abenov', M. B. Gabbassov², A. A. Ahmedov ${ }^{3, *}$ \\ Faculty of Mechanics and Mathematics, Al Farabi Kazakh National University, Kazakhstan \\ 2 Faculty of Mechanics and Mathematics, L.N. Gumilyiov Eurasian National University, st. Satbayev 2, Almaty district, Nur-Sultan 010000, \\ Kazakhstan \\ 3 Pusat Sains Matematik, Universiti Malaysia Pahang, Lebuhraya Tun Razak, 26300 Gambang, Kuantan, Pahang, Malaysia
}

\section{Article history:}

Received 2 August 2021

Received in revised form 5 December 2021

Accepted 11 December 2021

Available online 31 December 2021

\section{Keywords:}

Four-dimensional space; sphere; Euler model; Cauchy-Riemann conditions
The research deals with the stationary flow of an inhomogeneous incompressible fluid inside a spherical vessel under the influence of a potential mass force. Using the methods of four-dimensional analysis, the solution to the problem is constructed in an explicit analytical form. Exact solutions of the Euler equations for a homogeneous fluid are obtained only for some of the simplest problems. Researchers usually prove the existence and uniqueness of solutions to various initial - boundary value problems for Euler equations using the methods of a priori estimation. After that, the problem is usually solved by numerical methods. For an inhomogeneous fluid, when the unknown density is a variable, even obtaining a priori estimates becomes much more complicated, not to mention finding exact solutions. Nevertheless, in recent years, new methods of fourdimensional mathematics have been developed, giving previously unknown approaches to the study of nonlinear problems. In this paper, an exact analytical solution of the Euler equations describing the flow of an ideal inhomogeneous fluid inside a sphere is obtained. At the same time, the authors demonstrate new methods of four-dimensional analysis.

\section{Introduction}

A lot of scientific paper were devoted to the problems of fluid flow, and these problems were solved numerically [1,2]. In this work by using the methods of four-dimensional analysis an analytical solution of inhomogeneous fluid flow was investigated.

Nonstationary models of the flow of an ideal inhomogeneous fluid are given in the form of various initial-boundary value problems for a system of Euler equations of the form

$\rho\left[\frac{\partial \vec{V}}{\partial t}+(\vec{V} \times \nabla) \vec{V}\right]+\nabla P=\rho \vec{F}$

$\frac{\partial \rho}{\partial t}+\frac{\partial\left(\rho V_{1}\right)}{\partial x}+\frac{\partial\left(\rho V_{2}\right)}{\partial y}+\frac{\partial\left(\rho V_{3}\right)}{\partial z}=0$

\footnotetext{
${ }^{*}$ Corresponding author.

E-mail address: anvarjon@ump.edu.my
}

https://doi.org/10.37934/arfmts.90.2.5563 
where unknowns: $\rho(t, x, y, z), P(t, x, y, z)$ - the desired density and pressure of the liquid, $\vec{V}=$ $\left(V_{1}(t, x, y, z), V_{2}, V_{3}\right)$ - desired vector of the desired velocity vector of the fluid, and the mass force vector $\vec{F}=\left(F_{1}(t, x, y, z), F_{2}, F_{3}\right)$ are given [3-7].

When solving a specific applied problem, various initial and boundary conditions are added to the differential equations, Eq. (1) and Eq. (2). Note that this system of differential equations is nonlinear and difficult to study. The fact is that the system is four-dimensional in its essence because the unknown functions to be determined are real functions of four real variables: $t, x, y, z$. At the same time, to find classical solutions to various initial boundary value problems developed mathematical apparatus of one dimensional (theory of functions of a real variable) and two-dimensional analysis (theory of functions of a complex variable) is usually used. In this regard, researchers investigated the various simplifications of the Euler model.

It is not difficult to understand that a complete study of various initial boundary value problems for the general Euler model Eq. (1) and Eq. (2) requires a complete four - dimensional mathematical apparatus. A pioneering work in this direction is the monograph [8], which sets out the basics of fourdimensional analysis and describes specific, four-dimensional methods for studying such problems. For the latest results on the four dimensioanal mathematics see papers [9-15].

In this article, we will illustrate the application of the methods of four-dimensional analysis to the study of a rather complex problem of hydrodynamics, which has an important application value.

\section{Statement of the Problem}

\subsection{Formulation of the Main Result}

Let an inhomogeneous, ideal incompressible fluid, completely filled with a spherical vessel of radius $r$, move under the action of an external, stationary potential mass force. It is necessary to determine the hydrodynamic characteristics of such a flow. It is well known [9] that mathematically this problem is formulated as follows. In the domain $\Omega: x^{2}+y^{2}+z^{2}<r^{2}$ with the boundary $\mathrm{S}$ : $x^{2}+y^{2}+z^{2}=r^{2}$ it is required to find a bounded and sufficiently smooth solution of the boundary value problem for a system of Euler equations, of the following form

$$
\begin{aligned}
& \rho(x, y, z)\left(V_{1} \frac{\partial V_{1}}{\partial x}+V_{2} \frac{\partial V_{1}}{\partial y}+V_{3} \frac{\partial V_{1}}{\partial z}\right)+\frac{\partial P}{\partial x}=\rho(x, y, z) F_{1}(x, y, z) \\
& \rho(x, y, z)\left(V_{1} \frac{\partial V_{2}}{\partial x}+V_{2} \frac{\partial V_{2}}{\partial y}+V_{3} \frac{\partial V_{2}}{\partial z}\right)+\frac{\partial P}{\partial y}=\rho(x, y, z) F_{2}(x, y, z) \\
& \rho(x, y, z)\left(V_{1} \frac{\partial V_{3}}{\partial x}+V_{2} \frac{\partial V_{3}}{\partial y}+V_{3} \frac{\partial V_{3}}{\partial z}\right)+\frac{\partial P}{\partial z}=\rho(x, y, z) F_{3}(x, y, z)
\end{aligned}
$$

with continuity conditions

$$
\begin{aligned}
& \frac{\partial\left(\rho V_{1}\right)}{\partial x}+\frac{\partial\left(\rho V_{2}\right)}{\partial y}+\frac{\partial\left(\rho V_{3}\right)}{\partial z}=0 \\
& \frac{\partial V_{1}}{\partial x}+\frac{\partial V_{2}}{\partial y}+\frac{\partial V_{3}}{\partial z}=0
\end{aligned}
$$

and with the condition of non-flow through the border 
$\left.V_{n}\right|_{s}=0$

Here: $F_{i}(x, y, z), i=\overline{1,3}-$ components of a given, potential mass force, which we assume to be sufficiently smooth. In the future, we will clarify the conditions imposed on these functions. Additionally, for an ideal fluid, the equation of state is assumed to be known and is taken as

$\rho=\omega(P)$

We seek the classical solution of the problem Eq. (3) - Eq. (7)

$\rho(x, y, z), P(x, y, z), V_{1}(x, y, z), V_{2}, V_{3} \in C^{1}(\Omega) \cap C(\bar{\Omega})$.

Let $\vec{F}=\left(F_{1}(x, y, z), F_{2}, F_{3}\right)$ is potential force, then, $\exists Q(x, y, z) \in C^{1}(\Omega) \cap C(\bar{\Omega})$ such that, $\vec{F}=\nabla Q$. The scalar function $Q(x, y, z)$ which satisfies the conditions above is said to be potential of mass force.

\subsection{Main Theorem}

Let $c$ be a certain velocity characteristic of a given current. A function $\Phi(P)=\int_{P_{0}}^{P} \frac{d \tau}{\omega(\tau)}$ - the potential of mass power $Q(x, y, z)$ is an arbitrary solution of the differential equation

$\left(x^{2}+2 y^{2}+2 z^{2}-r^{2}\right) \frac{\partial Q}{\partial x}-x y \frac{\partial Q}{\partial y}-x z \frac{\partial Q}{\partial z}=\theta(x, y, z)$

where

$\theta=\frac{c^{2} x}{r^{4}}\left[2\left(x^{2}-r^{2}\right)\left(x^{2}+2 y^{2}+2 z^{2}-r^{2}\right)-\left(y^{2}+z^{2}\right)\left(2 y^{2}+2 z^{2}-r^{2}\right)\right]$

Then the problem Eq. (3) - Eq. (7) has a unique classical solution of the form

$$
\left\{\begin{array}{c}
V_{1}(x, y, z)=\frac{c\left(x^{2}+2 y^{2}+2 z^{2}-r^{2}\right)}{r^{2}} \\
V_{2}(x, y, z)=-\frac{c x y}{r^{2}} \\
V_{3}(x, y, z)=-\frac{c x z}{r^{2}} \\
\Phi(P)=Q(x, y, z)-\frac{c^{2}}{2 r^{4}}\left[x^{4}-\left(y^{2}+z^{2}\right)^{2}-r^{2}\left(2 x^{2}-y^{2}-z^{2}\right)\right] \\
\rho=\omega(P)
\end{array}\right.
$$

Note that the differential equation Eq. (8) has innumerable (continuum) smooth solutions $Q(x, y, z) \in C^{1}(\Omega) \cap C(\bar{\Omega})$. For each such solution, the mass force is determined $\vec{F}=\nabla Q$. By this we define a continuum of mass forces.

\section{Description of the Method for Solving the Problem}

We will rely on the theoretical foundations of four-dimensional analysis set out in [8]. Let $X=$ $\left(x_{0}, x_{1}, x_{2}, x_{3}\right) \in G \subset R^{4}-$ a dimensionless four-dimensional number. Consider all possible 
mappings of the form $U: G \rightarrow R^{4}$. With such mappings, each element $X=\left(x_{0}, x_{1}, x_{2}, x_{3}\right) \in G$ a welldefined image is matched - a four-component object of the following type

$U(X)=\left[U_{0}\left(x_{0}, x_{1}, x_{2}, x_{3}\right), U_{1}, U_{2}, U_{3}\right] \in R^{4}$

Definition 1. An object of the form $U=\left(U_{0}, U_{1}, U_{2}, U_{3}\right)$, which is an image obtained with some mapping $U: G \rightarrow R^{4}$, is called a four-dimensional function.

Definition 2. A four-dimensional function whose components are all real and continuous functions of four variables in the domain $G$ is called a continuous four-dimensional function, and the entire set of such functions is denoted as $C M(G)$. With respect to the operation of component-bycomponent addition and multiplication by a scalar, $C M(G)$ is a linear space.

Further, in [8], a description of a specific subset is given $M_{A}(G) \subset C M(G)$. Each element $M_{A}(G)$ It plays the role of a four-dimensional generalization of typical functions from real and complex analysis.

Definition 3. The element $u=\left(u_{0}, u_{1}, u_{2}, u_{3}\right) \in C M(G)$ is said to be a regular four-dimensional function in the domain $G \subset R^{4}$, if the relations (generalized Cauchy-Riemann conditions) of the following form are satisfied

$$
\begin{aligned}
& \frac{\partial u_{0}}{\partial x_{0}}=\frac{\partial u_{1}}{\partial x_{1}}=\frac{\partial u_{2}}{\partial x_{2}}=\frac{\partial u_{3}}{\partial x_{3}} \\
& \frac{\partial u_{0}}{\partial x_{1}}=\frac{\partial u_{1}}{\partial x_{0}}=\frac{\partial u_{2}}{\partial x_{3}}=\frac{\partial u_{3}}{\partial x_{2}} \\
& \frac{\partial u_{0}}{\partial x_{2}}=\frac{\partial u_{1}}{\partial x_{3}}=-\frac{\partial u_{2}}{\partial x_{0}}=-\frac{\partial u_{3}}{\partial x_{1}} \\
& \frac{\partial u_{0}}{\partial x_{3}}=\frac{\partial u_{1}}{\partial x_{2}}=-\frac{\partial u_{2}}{\partial x_{1}}=-\frac{\partial u_{3}}{\partial x_{0}}
\end{aligned}
$$

It is easy to check that the simplest, everywhere regular function is $u=X=\left(x_{0}, x_{1}, x_{2}, x_{3}\right)$. We denote the set of such functions by $M_{A}(G)$. The subset $M_{A}(G)$ of the linear space $C M(G)$ is also linear space with respect to the operations: component-by-component addition of elements and multiplication by a scalar. It is shown that [4], the sub linear space $M_{A}(G)$ infinite dimensional. It is proved that each regular function is a four-dimensional analog of the corresponding typical function from real and complex analysis.

Example 1. Consider a function $u=X^{2}=\left(u_{0}, u_{1}, u_{2}, u_{3}\right)$ of the four-dimensional independent variable $X=\left(x_{0}, x_{1}, x_{2}, x_{3}\right)$

$$
\begin{aligned}
& u_{0}\left(x_{0}, x_{1}, x_{2}, x_{3}\right)=2 x_{0} x_{1}-2 x_{2} x_{3} \\
& u_{1}\left(x_{0}, x_{1}, x_{2}, x_{3}\right)=x_{0}^{2}+x_{1}^{2}-x_{2}^{2}-x_{3}{ }^{2} \\
& u_{2}\left(x_{0}, x_{1}, x_{2}, x_{3}\right)=2 x_{1} x_{2}+2 x_{0} x_{3} \\
& u_{3}\left(x_{0}, x_{1}, x_{2}, x_{3}\right)=2 x_{1} x_{3}+2 x_{0} x_{2}
\end{aligned}
$$

It is easy to check that at the points of the form $X=\left(0, x_{1}, x_{2,0}\right)$ a function has a form

$$
u=X^{2}=\left(0, x_{1}{ }^{2}-x_{2}{ }^{2}, 2 x_{1} x_{2}, 0\right)
$$

which is just another form of representing an elementary function from a single complex variable

$$
u=z^{2}=\left(x_{1}+i x_{2}\right)^{2} .
$$


Similarly, in the points of the form $X=\left(0, x_{1,0,0}\right)$ we get a one-dimensional analog $U=X^{2}=$ $\left(0, x_{1}^{2}, 0,0\right)$, which is just another form of writing an ordinary function of a single real variable. This situation is characteristic of all regular four-dimensional functions, that is, the corresponding twodimensional and one-dimensional analogs are easily derived from its form (formula) in the specified way. For example, this is valid for a four-dimensional exponential function

$u=e^{X}=\left\{u_{0}\left(x_{0}, x_{1}, x_{2}, x_{3}\right), u_{1}, u_{2}, u_{3}\right\}$,

where

$$
\begin{aligned}
& u_{0}\left(x_{0}, x_{1}, x_{2}, x_{3}\right)=\frac{1}{2}\left[e^{x_{1}+x_{0}} \cos \left(x_{2}+x_{3}\right)-e^{x_{1}-x_{0}} \cos \left(x_{2}-x_{3}\right)\right] \\
& u_{1}\left(x_{0}, x_{1}, x_{2}, x_{3}\right)=\frac{1}{2}\left[e^{x_{1}+x_{0}} \cos \left(x_{2}+x_{3}\right)+e^{x_{1}-x_{0}} \cos \left(x_{2}-x_{3}\right)\right] \\
& u_{2}\left(x_{0}, x_{1}, x_{2}, x_{3}\right)=\frac{1}{2}\left[e^{x_{1}+x_{0}} \sin \left(x_{2}+x_{3}\right)+e^{x_{1}-x_{0}} \sin \left(x_{2}-x_{3}\right)\right] \\
& u_{3}\left(x_{0}, x_{1}, x_{2}, x_{3}\right)=\frac{1}{2}\left[e^{x_{1}+x_{0}} \sin \left(x_{2}+x_{3}\right)-e^{x_{1}-x_{0}} \sin \left(x_{2}-x_{3}\right)\right]
\end{aligned}
$$

Further, let $G$ be a compact set. Then $M_{A}(G)$ becomes an everywhere dense subset in the space $C M(G)$. And the latter contains all the required 4-vectors that occur in the applied problems of modern natural science. Therefore, it is sufficient to study applied problems in compact domains "in terms of" regular functions from $M_{A}(G)$. This is discussed in more detail in monograph [8]. Thus, one of the key results of the four-dimensional analysis is associated with the solution of a non-stationary equation of the form

$$
\frac{1}{c} \frac{\partial \theta(t, x, y, z)}{\partial t}+\frac{\partial u(t, x, y, z)}{\partial x}+\frac{\partial v(t, x, y, z)}{\partial y}+\frac{\partial w(t, x, y, z)}{\partial z}=0
$$

In the bounded domain $G \subset R^{4}$. Let $c$ - characteristic speed, $L$-characteristic measure (diameter) of the domain. We use notations

$x_{0}=\frac{c t}{L}, x_{1}=\frac{x}{L}, x_{2}=\frac{y}{L}, x_{3}=\frac{z}{L}$.

Definition 4. The following eight scalars: $\alpha_{k}, \beta_{k}, k=\overline{0,3}$ (real and complex numbers) are said to be the resolving parameters of the Eq. (11), if they are connected by the relations

$\alpha_{0} \beta_{0}+\alpha_{1} \beta_{1}-\alpha_{2} \beta_{2}-\alpha_{3} \beta_{3}=0$.

Auxiliary Theorem. Let $u=\left(u_{0}, u_{1}, u_{2}, u_{3}\right) \in M_{A}(G)$ - an arbitrary regular function, $\left(\alpha_{k}, \beta_{k}\right), k=\overline{0,3}$-an arbitrary list of the resolving parameters. Then the general solution of the (11) has the following form

$$
\begin{aligned}
& \theta(t, x, y, z)=\beta_{0} c u_{0}\left(\frac{\alpha_{0} c t}{L}, \frac{\alpha_{1} x}{L}, \frac{\alpha_{2} y}{L}, \frac{\alpha_{3} z}{L}\right) \\
& u(t, x, y, z)=\beta_{1} c u_{1}\left(\frac{\alpha_{0} c t}{L}, \frac{\alpha_{1} x}{L}, \frac{\alpha_{2} y}{L}, \frac{\alpha_{3} z}{L}\right) \\
& v(t, x, y, z)=-\beta_{2} c u_{2}\left(\frac{\alpha_{0} c t}{L}, \frac{\alpha_{1} x}{L}, \frac{\alpha_{2} y}{L}, \frac{\alpha_{3} z}{L}\right) \\
& w(t, x, y, z)=-\beta_{3} c u_{3}\left(\frac{\alpha_{0} c t}{L}, \frac{\alpha_{1} x}{L}, \frac{\alpha_{2} y}{L}, \frac{\alpha_{3} z}{L}\right)
\end{aligned}
$$


We refer the readers to [4] for the proof of the statement. Further, all our further actions will be related to the application of formula (12) to the simpler Eq. (5) in the original problem. As we will see later, it is this four-dimensional approach to the original task that leads us to achieve our goals.

\section{Proof of the Main Theorem}

First, we will separately consider Eq. (5) of the original problem.

$\frac{\partial V_{1}(x, y, z)}{\partial x}+\frac{\partial V_{2}(x, y, z)}{\partial y}+\frac{\partial V_{3}(x, y, z)}{\partial z}=0$

This equation is the stationary analog of the Eq. (11), when $\theta=0$, which can be achieved by choosing $\beta_{0}=0$, and the other functions do not depend on the time parameter, which is achieved by choosing $\alpha_{0}=0$. Thus, the general solution (13) will be as follows

$V_{1}(x, y, z)=\beta_{1} c u_{1}\left(0, \frac{\alpha_{1} x}{L}, \frac{\alpha_{2} y}{L}, \frac{\alpha_{3} z}{L}\right)$
$V_{2}(x, y, z)=-\beta_{2} c u_{2}\left(0, \frac{\alpha_{1} x}{L}, \frac{\alpha_{2} y}{L}, \frac{\alpha_{3} z}{L}\right)$
$V_{3}(x, y, z)=-\beta_{3} c u_{3}\left(0, \frac{\alpha_{1} x}{L}, \frac{\alpha_{2} y}{L}, \frac{\alpha_{3} z}{L}\right)$

where $\alpha_{1} \beta_{1}-\alpha_{2} \beta_{2}-\alpha_{3} \beta_{3}=0$.

Based on the results of the work [5], we will obtain

$u(X)=X^{2}-1 ; L=r ; \alpha_{1}=\beta_{1}=1 ; \alpha_{2}=\alpha_{3}=\sqrt{2} i ; \beta_{2}=\beta_{3}=-\frac{i \sqrt{2}}{4}$

Then, using the explicit form of the function components $u(X)=X^{2}-1$ (see Example 1 ) we have

$$
\begin{aligned}
& V_{1}(x, y, z)=\frac{c\left(x^{2}+2 y^{2}+2 z^{2}-r^{2}\right)}{r^{2}} \\
& V_{2}(x, y, z)=-\frac{c x y}{r^{2}} \\
& V_{3}(x, y, z)=-\frac{c x y}{r^{2}}
\end{aligned}
$$

It is easy to check that the functions (15) satisfy the conditions (5) and (6) of the original problem. Indeed, the execution of (5) is checked trivially, and

$\left.V_{n}\right|_{S}=\left.\left(2 x V_{1}+2 y V_{2}+2 z V_{3}\right)\right|_{S}=\left.\frac{2 c x}{r^{2}}\left(x^{2}+y^{2}+z^{2}-r^{2}\right)\right|_{S}=0$.

Now we write the first continuity Eq. (4) in the form

$\vec{V} \nabla \rho+\rho \operatorname{div} \vec{V}=0$

or taking into account Eq. (5), in the following form

$V_{1} \frac{\partial \rho}{\partial x}+V_{2} \frac{\partial \rho}{\partial y}+V_{3} \frac{\partial \rho}{\partial z}=0$ 
Thus, the desired density is $\rho(x, y, z)$ satisfies the Eq. (16). On the other hand, if $\rho=\omega(P)$ then

$\frac{\partial \rho}{\partial x}=\frac{\partial \omega(P)}{\partial P} \frac{\partial P}{\partial x} ; \frac{\partial \rho}{\partial y}=\frac{\partial \omega(P)}{\partial P} \frac{\partial P}{\partial y} ; \frac{\partial \rho}{\partial z}=\frac{\partial \omega(P)}{\partial P} \frac{\partial P}{\partial z}$

Substituting the density derivatives in Eq. (16), we get

$V_{1} \frac{\partial P}{\partial x}+V_{2} \frac{\partial P}{\partial y}+V_{3} \frac{\partial P}{\partial z}=0$

That is, the desired pressure satisfies equation (17), which is absolutely identical to Eq. (16). Further, using formulas Eq. (15), we can easily obtain

$V_{1} \frac{\partial V_{1}}{\partial x}+V_{2} \frac{\partial V_{1}}{\partial y}+V_{3} \frac{\partial V_{1}}{\partial z}=\frac{2 c^{2} x\left(x^{2}-r^{2}\right)}{r^{4}}$

$V_{1} \frac{\partial V_{2}}{\partial x}+V_{2} \frac{\partial V_{2}}{\partial y}+V_{3} \frac{\partial V_{2}}{\partial z}=-\frac{c^{2} y\left(2 y^{2}+2 z^{2}-r^{2}\right)}{r^{4}}$

$V_{1} \frac{\partial V_{3}}{\partial x}+V_{2} \frac{\partial V_{3}}{\partial y}+V_{3} \frac{\partial V_{3}}{\partial z}=-\frac{c^{2} z\left(2 y^{2}+2 z^{2}-r^{2}\right)}{r^{4}}$

Now the equations of motion (3) will be rewritten in the following form

$\left\{\begin{array}{c}\frac{1}{\rho} \frac{\partial P}{\partial x}=\frac{\partial Q}{\partial x}-\frac{2 c^{2} x\left(x^{2}-r^{2}\right)}{r^{4}} \\ \frac{1}{\rho} \frac{\partial P}{\partial y}=\frac{\partial Q}{\partial y}+\frac{c^{2} y\left(2 y^{2}+2 z^{2}-r^{2}\right)}{r^{4}} \\ \frac{1}{\rho} \frac{\partial P}{\partial z}=\frac{\partial Q}{\partial z}+\frac{c^{2} z\left(2 y^{2}+2 z^{2}-r^{2}\right)}{r^{4}}\end{array}\right.$

In general, the system Eq. (18) is solvable for any potential of mass forces $Q(x, y, z)$. But we have to choose it so that Eq. (17) is additionally satisfied, which is equivalent to fulfilling Eq. (16). That is, the condition for unambiguous determination of pressure (density) is a condition of the form

$V_{1}\left[\frac{\partial Q}{\partial x}-\frac{2 c^{2} x\left(x^{2}-r^{2}\right)}{r^{4}}\right]+V_{2}\left[\frac{\partial Q}{\partial y}+\frac{c^{2} y\left(2 y^{2}+2 z^{2}-r^{2}\right)}{r^{4}}\right]+V_{3}\left[\frac{\partial Q}{\partial z}+\frac{c^{2} z\left(2 y^{2}+2 z^{2}-r^{2}\right)}{r^{4}}\right]=0$

From here, after opening the brackets, we come to Eq. (8). If the potential $Q(x, y, z)$ is chosen as the solution to Eq. (8), then Eq. (4) of the original system will be automatically fulfilled. Further, if

$\Phi(P)=\int_{P_{0}}^{P} \frac{d \tau}{\omega(\tau)} \Rightarrow \nabla \Phi(P)=\frac{1}{\rho} \nabla P$

Therefore, system Eq. (18) has a unique solution of the form

$\Phi(P)=Q(x, y, z)-\frac{c^{2}}{2 r^{4}}\left[x^{4}-\left(y^{2}+z^{2}\right)^{2}-r^{2}\left(2 x^{2}-y^{2}-z^{2}\right)\right]=\Psi(x, y, z)$

From Eq. (19) we determine the pressure $P(x, y, z)$. After that, we will also find the exact density formula of the form $\rho=\omega(P)$. The proof of the main theorem is completed. 


\section{Conclusion}

We were able to obtain an explicit analytical solution to a nonlinear system of differential equations. Obviously, this fact emphasizes the attractiveness of the mathematical apparatus of fourdimensional mathematics in solving applied problems of this kind. Next, we could take the equation of state in its simplest form (the Mendeleev-Clapeyron formula), namely

$\rho=\omega(P)=\frac{P}{R_{g} T}$

where $R_{g}$ - universal gas constant, and $T$ - constant value, the temperature of the liquid. In this case, the formula (19) will be rewritten as follows

$\frac{\Phi(P)=R_{g} T \ln P}{P_{0}}=\Psi(x, y, z)$

From here we obtain

$P(x, y, z)=P(0,0,0)+\exp \left[\frac{\Psi(x, y, z)}{R_{g} T}\right]$

Unknown number $P(0,0,0)$ now it is determined from the condition of the form

$\int_{\Omega} P(x, y, z) d x d y d z=0$

After that, the density is also uniquely determined $\rho=\frac{P}{R_{g} T}$.

\section{References}

[1] Sidik, Nor Azwadi Che, Solihin Musa, Siti Nurul Akmal Yusof, and Erdiwansyah Erdiwansyah. "Analysis of Internal Flow in Bag Filter by Different Inlet Angle." Journal of Advanced Research in Numerical Heat Transfer 3, no. 1 (2020): 12-24.

[2] Wei, Ong Yong, and Siti Ujila Masuri. "Computational fluid dynamics analysis on single leak and double leaks subsea pipeline leakage." CFD Letters 11, no. 2 (2019): 95-107.

[3] Arfken, George B., and Hans J. Weber. "Mathematical methods for physicists." Academic Press, (1999): $165-169$. https://doi.org/10.1119/1.19217

[4] Batchelor, Cx K., and G. K. Batchelor. An introduction to fluid dynamics. Cambridge university press, 2000. https://doi.org/10.1017/CBO9780511800955

[5] L. G. Loitzansky. "Fluid Mechanics, 2nd edition." 2003.

[6] Jabar, Siti Norbakyah, and Salisa Abdul Rahman. "A Comparative Study on Components Sizing for Conventional Boat and Pherb Powertrains using Water Driving Cycle." Journal of Advanced Research in Applied Sciences and Engineering Technology 16, no. 1 (2019): 41-48.

[7] Sukamta, Sukamta. "Computational Fluid Dynamics (CFD) and Experimental study of Two-Phase Flow Patterns GasLiquid with Low Viscosity in a Horizontal Capillary Pipe." CFD Letters 11, no. 8 (2019): 16-23.

[8] M. M. Abenov. "Four-dimensional mathematics: methods and applications." Almaty, Kazakh University Press (2019).

[9] Abenov, M. M., M. B. Gabbassov, and F. Y. Ismagulova. "Movement of fluid inside the sphere." International Journal of Engineering \& Technology 7, no. 4 (2018): 42-44. https://doi.org/10.14419/ijet.v7i4.30.22001 
[10] M.M.Abenov. "Some applications of the spectral theory of the function of a complex variable." Scientific Monograph 2013.

[11] M.M.Abenov. "Solutions of the system of Navier-Stokes equations." Scientific Monograph 2013

[12] M.M.Abenov. "Exact solutions of the three-dimentional Navier-Stokes equations."-V Congress of the Turkic world mathematicians 2014.

[13] M.M.Abenov. "On classes of exact solutions of the continuity equation." Proceedings of the All-Russian Conference Nonlinear Waves: Theory and new applications 2016.

[14] M.M.Abenov. "On the continuum of exact solutions of the Euler system." Proceedings of the Ufa International Mathematical Conference 2016.

[15] M.M.Abenov. "On exact solutions of the basic Navier-Stokes model." Proceedings of the International Mathematical Conference dedicated to the 100th anniversary of Academician A.D.Taimanov 2017. 